\title{
A new study for the global asymptotic stability of a general predator-prey model
}

\author{
Manh Tuan Hoang ${ }^{1}$ \\ ${ }^{1}$ FPT University
}

August 2, 2021

\begin{abstract}
In a previous paper [L. M. Ladino, E. I. Sabogal, Jose C. Valverde, General functional response and recruitment in a predatorprey system with capture on both species, Math. Methods Appl. Sci. 38(2015) 2876-2887], a mathematical model for a predator-prey model with general functional response and recruitment including capture on both species was formulated and analyzed. However, the global asymptotic stability (GAS) of this model was only partially resolved. In the present paper, we provide a rigorously mathematical analysis for the complete GAS of the predator-prey model. By using the Lyapunov stability theory in combination with some nonstandard techniques of mathematical analysis for dynamical systems, the GAS of equilibria of the model is determined fully. The obtained results not only provide an important improvement for the population dynamics of the predator-prey model but also can be extended to study its modified versions in the context of fractional-order derivatives. The theoretical results are supported and illustrated by a set of numerical examples.
\end{abstract}

\section{Hosted file}

Predator-Prey model_new.pdf available at https://authorea.com/users/428704/articles/532498-anew-study-for-the-global-asymptotic-stability-of-a-general-predator-prey-model 